\title{
Intraoperative X-rays during coccygectomy
}

\author{
Patrick M. Foye ${ }^{1} \cdot$ Gerard J. D'Onofrio ${ }^{1}$
}

Accepted: 24 March 2018 / Published online: 3 April 2018

c) Springer-Verlag GmbH Germany, part of Springer Nature 2018

I commend your journal and authors Graves et al. on the excellent and educational publication titled: "Use of intraoperative lateral pelvic X-ray to localize and ensure coccyx removal during sacrococcygeal teratoma resection." [1].

The article provides a logical and strongly compelling case for use of a simple lateral radiograph within the operating room immediately before coccygectomy. This radiograph confirms the location of the sacrococcygeal junction, and thus confirms the upper border of the surgical target site for complete coccygectomy. There are many reasons why this approach makes perfect sense. There are formidable challenges in discerning the sacrococcygeal junction solely by surgical visualization, given the limited area of surgical exposure, relatively small field of view, and substantial variability in coccygeal anatomy. Similarly, the article convincingly argues for a second intraoperative X-ray at the end of the surgery to confirm that the coccygectomy was complete rather than partial.

While the authors use intraoperative X-rays for tumorrelated coccygectomy, we would be extremely interested in whether they would agree that such X-rays would also be helpful immediately before/after coccygectomies unrelated to tumors. At our Coccyx Pain Center, we see many patients who have persistent coccydynia (tailbone pain) despite coccygectomy. Subsequent imaging often reveals inadvertently retained coccygeal fragments, as well as pointy (and painful) edges at the osteotomy site, etc. Using the approach of Graves et al. might help patients avoid such unfavorable outcomes.

Funding None.

\section{Compliance with ethical standards}

Conflict of interest Author Dr. Patrick M. Foye declares that he has no conflict of interest. Author Mr. Gerard J. D'Onofrio declares that he has no conflict of interest.

Ethical approval This article does not contain any studies with human participants or animals performed by any of the authors.

\section{Reference}

1. Graves CE, Idowu O, Zovickian J, Pang D, Kim S (2017) Use of intraoperative lateral pelvic X-ray to localize and ensure coccyx removal during sacrococcygeal teratoma resection. Pediatr Surg Int 33(3):389-392
This comment refers to the article available at https://doi. org/10.1007/s00383-016-4025-8.

Patrick M. Foye

doctor.foye@gmail.com; patrick.foye@ rutgers.edu

Gerard J. D'Onofrio

gerard.donofrio27@gmail.com

1 Department of Physical Medicine and Rehabilitation, Coccyx Pain Center, Rutgers New Jersey Medical School, 90 Bergen St, D.O.C. Suite 3100, Newark, NJ 07103-2425, USA 